\title{
NotAS LÉXICAS SOBRE EL ARAGONÉS. A PROPÓSITO DE LA TRADUCCIÓN DE LA AGRICULTURA DE PALLADIO AL CASTELLANO
}

\author{
José Antonio Pascual \\ Real Academia Española \\ joseapascual@yahoo.es
}

Resumen: Dejando a un lado el ingente número de catalanismos presentes en la traducción al castellano del De re rustica de Palladio, atribuida a Ferrer Saiol, en el presente trabajo se analizan algunos de los también muchos aragonesismos que la pueblan. En concreto, se presenta un estudio sistemático de once de ellos: conrear, empeltar, borró borrons, senalla, lambrusca, brocada, entrecavar, palafangar, pámpano, espleyto y espleytar. Algunas son formas que el catalán compartía con el aragonés, pero no con el castellano, donde tampoco tuvieron un amplio recorrido. No obstante, la importancia del aragonés no hemos de medirla solo por los aragonesismos que se introdujeron en castellano, sino también por el hecho previo de que no dudaran los traductores en servirse de ellos, a sabiendas, incluso, de que muchos no iban a pervivir. Es la prueba de una actitud abierta hacia las lenguas de la Corona de Aragón.

Palabras clave: Palladio, Agricultura, traducción, léxico castellano, aragonesismos.
Title: Lexical notes on Aragonese. Regarding the translation of Palladio's Agricultura into Spanish.

Abstract: Leaving aside the huge numbre of catalanisms present in the Spanish translation of Palladio's De re rustica, atributed to Ferrer Saiol, in this paper some of the many aragonesisms that populate it are also analyzed. Specifically, a systematic study of eleven of them is presented: conrear, empeltar, borró borrons, senalla, lambrusca, brocada, entrecavar, palafangar, pámpano, espleyto and espleytar. Some are forms that Catalan shared with Aragonese, but not with Castilian, where they did not have a long life either. However, the importance of Aragonese should not be measured only by the aragonesisms that were introduced into Spanish, but also by the fact that the translators did not hesitate to use them, even knowing that many were not going to survive. It is proof of an open attitude towards the languages of the Crown of Aragon.

Keywords: Palladio, Agricultura, translation, Spanish lexicon, aragonesisms. 
La comparación entre el léxico de los distintos textos de un corpus que podemos consultar los filólogos, así como la mayor accesibilidad a las obras de otras lenguas y de la nuestra, han hecho que de un tiempo a esta parte hayan cambiado las posibilidades de estudio de la historia del léxico español. Pero para llegar a esta situación no ha bastado con los avances de índole técnica que se han dado en el campo de la filología; ha sido necesario también que se produjera un cambio en la mentalidad de las personas que median entre los textos y el trabajo filológico: bibliotecarios, archiveros y hasta algunos filólogos también. Sin esa nueva normalidad (por utilizar un sintagma mal empleado, pero propio de estos tiempos), en la que ese tipo de actitudes antiguas es hoy la excepción, ni siquiera se me hubiera ocurrido pensar en escribir estas notas. El hecho es que parece hoy normal que la Biblioteca Nacional de España ponga en la red, a disposición de todo el mundo, una serie de códices que pudieran tener algún interés para el trabajo filológico: el MS 10211 de la Agricultura de Palladio es uno de ellos, que he podido consultar cómodamente desde mi ordenador cuando he necesitado hacerlo. Igual que gracias al Servici de Patrimoni Històric i Artístic Tècnico del Ajuntament de València he dispuesto igualmente (a lo que no han sido ajenas la profesionalidad y amabilidad de D. ${ }^{a}$ Itziar Vilar Rey) de una reproducción del MS 6437 del fondo Serrano Morales del Arxiu Municipal, manuscrito que otrora «una verdadera conspiración de silencios impidió que algunos filólogos lo consultaran» (Hauf 1989: $384, \mathrm{n} .1$ ). A tal proceder de estos servicios bibliotecarios y archivísticos, se añade que distintos grupos de investigadores han sabido entender la importancia que tiene desarrollar un trabajo básico que nos permita a los demás disponer de recursos que se han convertido en imprescindibles para nuestras tareas, como lo han sido ahora para mí el Corpus del Nuevo Diccionario Histórico del Español (en adelante CDH) y el Fichero General de la Academia, accesibles ambos online, que permiten contrastar los datos que contiene el texto castellano de Palladio con los de muchos otros textos de nuestra lengua; el Diccionari del castellà del segle XV a la Corona d'Aragó, dirigido por C. Lleal (en adelante DICCAXV), accesible también online, que centra esa comparación en los textos aragoneses; la Biblioteca Digital de Textos del Español Antiguo, de The Hispanic Seminary of Medieval Studies (HSMA) de Madison, que desde los años 70, en papel, microficha o soporte digital, viene proporcionando unos recursos importantes para la investigación filológica (en este caso por medio del acceso online a la excelente transcripción de Th. M. Capuano de la Agricultura de Palladio ${ }^{1}$ ); y, por supuesto, el fondo

\footnotetext{
Se trata del MS 10211 de la Biblioteca Nacional de España (en adelante, BNE), que acoge, además de esta obra, otras traducidas también del catalán, con toda probabilidad en algún lugar de la Corona de Aragón (Cifuentes: <Sciència.cat DB MS 1363 (29/06/2020>), que citaremos aquí: el Tratado de plantar o enjerir árboles o de conservar el vino, ff. 224r-244r, que es una traducción del De plantationibus arborum et de conservatione vini de Gottfried de Franconia (Martí Escayol 2009: 133, n.
} 
tan impresionante de textos que facilita Google en la red. En la presente ocasión me he apoyado además en la amistad de Ignacio Sotelo, quien hace tiempo, con gran generosidad, me entregó su cuidadosa edición de la traducción castellana de Palladio, llevada a cabo hace ya medio siglo y que lamentablemente ha permanecido inédita, así como otros materiales de mucho interés relacionados con esta traducción.

\section{INTRODUCCIón}

Entrando en mis notas léxicas a este texto, he querido dejar de lado la cantidad ingente de catalanismos volcados en él (vid. Martínez Romero 2008: 114 y 115) para ocuparme de un reducidísimo número de aragonesismos, entre los muchos que aparecen en esta traducción, hecha por una persona que se tenía que manejar bien en catalán y aragonés —idea en la que coincido totalmente con Butiñá (1996: 215, 216), Martínez Romero (2008: 113) y con varios filólogos más que cita Sebastián Torres (2014: 74), igual que coincido con ellos en que esa persona no fue Ferrer Sayol, algo que J. Butiñá ha argumentado de una manera convincente- En esas condiciones no es extraño que se recurriera en esta versión castellana — unas veces consciente y otras inconscientemente- a algunas formas que el catalán compartía con el aragonés, pero no con el castellano. Es lo que trataré de mostrar, aunque me apresuro a añadir que la mayor parte de esos aragonesismos ha tenido poco recorrido en la historia del español, salvo en algunas de sus variedades orientales, relacionadas con el aragonés.

Estamos ante una traducción, que como otras de la época, buscaba sencillamente hacer comprensible el texto traducido, que luego podría ir mejorando poco a poco en las copias posteriores que se hicieran de él. En esas copias, sin dárseles el carácter de una nueva versión, se podrían realizar «en mayor o menor medida una labor similar con las traducciones, que [se] revisan mientras [se] copian» (Morrás 2002: 35). Es lo que ocurre con los dos manuscritos que contienen la traducción del Árbol de las batallas, encargada por el marqués de Santillana, en el que el copista del ms. 10103 trató de desprenderse de bastantes aragonesismos contenidos en el ms. 19 102, para acercar de ese modo esta versión al tipo de lengua elaborada del castellano del siglo XV que varios intelectuales aragoneses tomaron como modelo

15) y la Memoria de las labranzas, de plantar y de sembrar y de pensar quése debe hacer para toda hortali$z$ a, la cual es presa de los mejores hortelanos de Barcelona, que ocupa los ff. $211 \mathrm{v}-214 \mathrm{r}$ de ese manuscrito. La fecha que aparece en el $C D H$ para estas obras es 1385, pero de lo único que tenemos seguridad es de la fecha de la copia del manuscrito, de mediados del siglo XV. Ambos textos están incluidos en el $C D H$ y el segundo en el DICCA XV, con la abreviatura Labores. 
(Pascual 2018: 648). Esta traducción contenida en el manuscrito 10211 de la BNE por el que ahora me muevo hubiera sido un buen candidato para que un copista posterior hubiera dejado de lado un buen número de sus orientalismos.

\section{CONREAR 'CULTIVAR'}

Estos son los ejemplos de conrear 'cultivar' que he encontrado en la traducción:

Traduciendo al cat. conrear: «personas que labren e conrreen la tierra», f. 1r; «agricultura e lauor que es conrrear la tierra , f. $3 \mathrm{r}$; « fazer conrrear las tierras e obrar obras de aquellas», f. 3v; «conrrear e labrar», f. 8v; «han a conrrear ponçineros e limoneros», f. 42v; «la su manera de conrrear», f. 99v.

Traduciendo al cat. culturar: «fazian labrar e conrrear sus tierras», f. $3 \mathrm{v}[\ll 1 l a u-$ raven e fahien llaurar e culturar lurs terres», f. 2 r $]^{2}$.

Sin correlato en el f. $42 \mathrm{v}$ del manuscrito catalán, donde le correspondía que apareciera la voz que se ha traducido: «non han menester de otra manera de conrrear njn ayuda $\gg$, f. $82 \mathrm{v}$.

Añádanse los siguientes ejemplos, aragoneses también, con algunos sentidos más, tomados del DICCAXV (s. v. conrear):

«mesmo toue vn tiempo vn pequeño huerto cabe el monesterio en el qual conreauamos hortalizas e vn gentil de noche entro e furto las», García de Santa María, Vida de santos (c1488), f. 15v.

«ciudat o la mayor partida de aquellos e pocos de los ditos tenjan conrreados aptos et sufficientes a moler e los mas finquauan distitujdos et inhabiles», $\mathrm{Or}$ dinaciones (1429), f. $24 \mathrm{v}$.

«la dita ciudat e sus terminos sian tenidos tener aquellos aptos sufficientes e conrreados de buenas mitelas ruedas e otras cosas necessarjas pora moler», Ordinaciones (1429), f. 25 r.

«colora o de sangre o de otro vmor e quando natura non puede correar el dicho vmor desordenado lexalo asi commo aboresçido», Recetario de Gilberto (1471), f. 53r.

2 La referencia a los ejemplos del manuscrito catalán, que no pudo ser la base directa de la traducción de la Agricultura de Palladio, pues es posterior a la propia traducción, la encierro entre corchetes y cito los folios por la numeración a lápiz que aparece en su parte superior derecha. 
«todas vuestras cortesias entiendo de dar fin. E por quanto aqueste apetito conrreado que con fuerça e razon ensemble ayusta e mueue aquellos a compassion», Cancionero de Coimbra (1448-65), f. 102v, en un poema del poeta catalán Pere Torroella.

Conrear se extiende por un amplio espacio del que está excluido el castellano: lo ocupan el occ. ant. conrezar 'équiper, corroyer' (Levy 1920:s. v.), el fr. ant. correer, luego corroyer 'preparer une matière en la battant, en l'étirant, en la foulant' (TLF: s. v. corroyer) y el it. corredare 'fornire di tutto ciò che è necessario o utile' (TLIO: $s$. v.), aparte, claro está, del cat. conrear referido a 'preparar las tierras para su cultivo'.

Contando con la información del $C D H^{3}$, a los ejemplos de la traducción de la Agricultura se añaden en ese corpus varios aragoneses más, como los de la Grant crónica de Fernández de Heredia (1385): «conrrearon e lauraron», de la traducción de De las ilustres mujeres (1494): «mando plantar e conrear», y de la traducción del Tirante el blanco (1511): «el ortolano tenía su herramienta para conrear el huerto y para su dormir». Por su parte Corominas (DECH: s. v. arrear, DECAT: s. v. conrear), que atribuye a conrear la condición de catalanismo, proporciona un ejemplo del Espejo de la vida humana, de Sánchez de Arévalo (1491), libr. 1, cap. 22, f. 38r, tomado del Diccionario de Autoridades ${ }^{4}$ « En la qual [la tierra] labrar e conrear quantos trabajos, quantos cuydados del cuerpo y del pensamiento los hombres suffren, ninguno lo sabe sino el que labra la tierra»; se trata de un escritor que, como Santillana, Mena o Gómez Manrique, no tenía empacho en servirse de voces orientales 5 . En el sur, en Murcia, tenemos en la actualidad conrearse y conreo, registrados por García Soriano (1980: xlvii).

3 Coloco entre paréntesis la fecha de los textos que aparece en el propio corpus de los textos, si bien en ocasiones la sustituyo por otra, citando de dónde la tomo.

4 Erróneamente da a esta palabra el significado de 'binar, dar segunda reja', que llega hasta el $D L E$. Esta acepción está originada por una mala interpretación de la geminación «labrar y conrear», que, como muestran los ejemplos que acabo de citar, se emplean como sinónimos, no como verbos de significado distinto.

5 Y ello, no solo por ciertos rasgos gráficos que aparecen en esta obra, como la representación de la [n] por ny o de la secuencia [ka] por qua, sino también por usos como vendema (Domene Verdú 2010: 7, cita el aragonesismo venema en el habla de Villena [Alicante], para los casos aragoneses con pérdida de la yod; el $D E C H$, s. v., cita un venemar de un documento de 1371) o touidos, f. 36v, que aparece abundantemente en los incunables impresos en Aragón. Si estos casos pudieran proceder del copista, hay otros que parecen propios del escritor, como pelayría: «La arte de la lana, siquier pelayria», f. 40r (vid.DECH: $s$. 
Estos datos permiten matizar ahora la idea de Corominas atribuyendo a conrear la condición de aragonesismo, aunque se hubiera tomado en préstamo del catalán.

\section{EMPELTAR 'INJERTAR'}

Lo normal es que se recurra a enxerir para traducir las voces catalanas enxerir y empeltar. No obstante, en tres ocasiones se mantiene la misma palabra en la traducción del cat. empeltar: «enpeltauan o enxirian», f. 2 r [cat. «empeltavan», f. 2r]; «empeltar», ff. 65v, 66r (2 veces) [cat. empeltar, f. 34r (2 veces)]. Tenemos, por otro lado, el sust. empelto: «enxierto o empelt», f. 73r [cat. «l'empelt», f. 37v].

Las palabras catalanas empeltar y empelt aparecen en esa área continua del sur de la Galia ocupada por el occitano — de su variedad bearnesa pasó al euskera de la Soule: enpheltü 'empelt', enphéltat 'empeltar'; también mentatu 'injertar' (Agud \& Tovar 1994: 921) - y por el catalán; de ahí pudo tomarse en aragonés, donde aparece empeltre 'olivo injertado' (Borao: s. v. empeltre; Buesa 1955: 71; DECAT: s. $v$. empeltar; vid. empeltre en el cat. ribagorzano y en el de la Llitera). Se extiende al sur, por esa área lingüística, a caballo entre Castilla y Aragón, que estudió Diego Catalán, en «De Nájera a Salobreña» (vid. Llorente 1991: 168, 169), llegando al murciano empeltar (DECAT: s. v. empeltar; Llatas 1959: s. v. empeltar; Torreblanca 1976: s. vv. empelte y empeltador) y al andaluz oriental.

Como en el caso anterior estamos ante un aragonesismo que había sido tomado a su vez en préstamo del catalán.

\section{BORRó BORRONS 'BROTE DE UNA PLANTA'}

Para referirse al brote de una planta, el castellano, aparte de esa y de varias voces más (ojo [cat. ull], nudo [cat. nus] o botón [cat. botó], ha contado con yema ${ }^{6}$, y el catalán, por su parte, con borró:

v.parar, para la explicación de pelaire como catalanismo. De pelairía, que solo se encuentra citada ahí, $D E C A T$ : s. v. parar, muestra ejemplos que permiten entender la procedencia de esta voz: «Que negú catalá no gaus comprar draps cruds en les pereyries, $c 1306$; etc.); «obra buydada», f. 40v (DECH: $s$. v. buido; DECAT: s. v. buit: «Buidat, -ada: "vaxella buydada: vasa conflatilia", "imàgens buydades: imagines conflátiles”»). Del mismo modo que tenemos un atraçar 'perseguir': «el dyablo nos atraça e procura juegos porque no lloremos», f. 48r, que se ha debido tomar del occitano o catalán (vid. $D E C H$ : s. v. traer, y DECAT: s. v. traure).

6 Yema ha tenido en castellano más vigor que en catalán, donde quedó muy pronto anticuada (DECH: s. v. yema, y DECAT: s. v. gemat; DCVB: s. v. gema). 
«... por tal que el agua que salira del sarmjento non destruya la yema el borro», f. 12 r [cat. «... per tal laygua que exira del sarment no destroeixca lo borro o la gema», f. 6r].

«la borra ${ }^{7}$ de la çepa o del sarmiento», f. 12v [cat. «lo borro del çep o de la sarment $\gg$, f. $6 \mathrm{v}]$.

«non tajes de la part del borro o de la yema njn mucho çerca de aquel», f. 69v [cat. «no talles de la part del borro ni massa prop de aquell», f. 36r].

«el agua que sale non faga dañyo al borro», f. 69v [cat. «laygua quen hix non fassa dapnatge al borro», f. 36r]. «dos yemas o borrons», f. 71r [cat. «.ij. borrons», f. 36v].

En el mismo MS 10211 de la BNE en que está la traducción de la Agricultura, tenemos el siguiente ejemplo, que tomo del DICCA XV: «Los enxiertos deuen ser bien espesos de borrons», Labores, f. 214r; está también en otro texto de ese mismo manuscrito, que se incluye en el $C D H$, el Tratado de plantar: «yemas njn borrones», «yemas o borrones», f. 232v.

Los datos aragoneses tomados del $A L E A R N$ han permitido a Llorente (1991: 169) explicar esta palabra como debida a la penetración de la voz, desde el catalán a la parte oriental del territorio de habla aragonesa, donde encontramos para la designación de la yema de la vid: borra, borrón y borró, frente al territorio situado al oeste, en que lo que aparece es brotón, brotó, yema y chema. Al sur disponemos de información que pertenece a ese espacio que cité antes, que está a caballo entre Castilla y Aragón y, pasando por Albacete y Murcia, llega a la Andalucía oriental, aparte de territorios castellanohablantes de Valencia y Alicante, donde podría haber penetrado el catalanismo desde el antiguo reino de Valencia. Es la idea de Coromines (DECAT: s. v. borra, n. 19): «A Murcia, on s'ha pres borrón del català, també es degué formar un regressiu així, i després el verb borrar (García Soriano) i desborrar «quitar a los árboles, y con particularidad a las moreras pequeñas, los cogollos o tallos que arrojan por el tronco» (Cabrera, Dicc. de Etimologías [p. 235, s. v. desborrar]). Tanmateix aquest verb no deu ser recent [en català] ni merament local, car segons AlcM s'usa també en el Camp de Tarragona i ja n'hi ha un cas de 1410 en un sermó de St. Vicent Ferrer (veg. AlcM)». El hecho es que distintas formas de borrón 'yema' y sus variantes (gorrón, por ejemplo) y derivados (es el caso de borrar 'brotar', emborronar, engorronar, desborrar, desborronar 'quitar a las moreras los tallos del tronco' y borradura 'sarpullido') se registran en Albacete, Murcia y Andalucía Oriental (además de R. Cabrera 1837, cit. por Coromines, vid. Sevilla 1919: s. vv.

El caso de borra quizá se explique por haber sido atraída por el cast. borra; está por borró, como muestra la concordancia: «la borra de la çepa o del sarmjento es çerrado», 12v. 
borrar, borrón, borradura [en sentido figurado 'sarpullido'], desborrar y desborronar; Llatas 1959: 137; Torreblanca 1976: 229-230; e Idáñez 2015: 417).

Este borró 'brote' y su derivado borronar 'brotar', que aparecen en nuestro texto, podemos considerarlos préstamos del catalán al aragonés.

\section{SENALLA 'CUÉvano'}

Combinar en el estudio filológico la cronología de las palabras con su distribución en el espacio puede originar algunas contradicciones, si bien tratar de solucionarlas contribuye a poder entender mejor las cosas. Así, el DECAT (s. v. peu) registra polzim 'pezón, rabito delgado y corto que une el fruto a la planta' a finales del siglo XV, pero propio solo de Valencia; la voz abunda en el texto catalán y hasta aparece en el castellano traduciendo en un par de ocasiones al val. polzim y en otra al cat.pampol.

Contrasta esta situación con la de senalla, cognado del cast. cenacho, que se repite varias veces (acompañada de su sinónimo cuévano) en un pasaje de la traducción de la Agricultura:

Toma vn cueuano de mjmbres o vna senalla. E por medio del suelo del cueuano o por el fondon faz pasar vn sarmjento [...] e finche de tierra el cueuano o senalla [...] dentro de vn añyo echara rrayzes dentro del cueuano [...], E syn dubda puedes lo trasplantar e non ayas dubda que se muera pues lo fazes plantar con el cueuano o senalla do avra echado rrayzes, f. $67 \mathrm{v}$.

En el manuscrito catalán no aparece senalla en el folio del que se traduce el texto anterior (f. 35v), ni tampoco recuerdo haberla encontrado en algún lugar del códice (aunque no pondría la mano en el fuego de que no aparezca en él). Estaríamos, a diferencia de lo que ocurría con polzim, con una forma del catalán norteño, si atendemos a la explicación del DECAT (s. v. senalla): «En terres valencianes escasseja o desapareix l'ús del mot [...]; no en tinc altra noticia valenciana que la d'un masculí senall en Escrig, 1851: "especie de cesto de mimbres; senall en tapa: excusabaraja”, que precisament amb aquest gènere masculí (exclusivament d'allà) revela la influència dominant del masculí mossàrab senatxo».

No me atrevería a ir más lejos tratando de encontrar una explicación a esta contradicción a que asistimos en el texto catalán, ni aun cuando dispusiera de muchos datos más, por la poca seguridad con que me muevo por estos terrenos; pero esos datos sí me ayudarían a sentirme un poco más seguro en el ámbito del castellano, 
en que pienso - con toda provisionalidad - que con senalla podríamos estar ante una voz que el traductor, si fuera valenciano, la desconociera en catalán, pero podría constarle su existencia en aragonés, de forma que en este caso para «trobar els mots exactes i més adients» (Hauf 1989: 383) en la traducción pudo haber recurrido al aragonés. El problema es que a este respecto mis datos se reducen a los que proporciona, por una parte, el $C D H$, que, junto a estos tres ejemplos, incluye el siguiente del Chronicon Mundi, traducido al aragonés, a instancias de Fernández de Heredia, acompañada también por el sinónimo canasta: «Ella, uidient la senalla o canasta »; por otra, en el Fichero General de la Academia tenemos tres cédulas que nos proporcionan un ejemplo del aragonés de Ayerbe (Buesa 1955: 76), al que acompaña también el más general, cuévano (ibid.: 78); a la vez que una referencia a Fort Cañellas (1988: 834), que no he podido comprobar, y la indicación de que aparece en el ALEANR, en Huesca.

Con estos datos pienso que la situación de senalla en nuestra traducción correspondería a la de los aragonesismos que hemos ido viendo hasta aquí, tomados en préstamo del catalán.

\section{LAMBRUSCA 'UVA SILVESTRE'}

El cast. lambrusca es la traducción del cat. llambrusca, continuador del lat. labrusca 'uva sivestre' (DECAT: s. v.). No contaba el traductor con una palabra castellana para designar 'las uvas silvestres o agrestes', por lo que le venía bien, de consuno con el original, mantener, junto a la palabra catalana, los rodeos con que se podían designar estas uvas: «Çepas saluajes que hombre llama lambrunscas», f. 8v [cat. «çeps saluatges ques dien lambrusques», f. 4v]; «Los sarmjentos negros Conujene a saber las lambruscas», f. 12r [cat. «sarments negres o ambrusques» (f. 6v)]; «las vuas agrestas ${ }^{8}$ o saluages que se dizen lambruscas », f. 154v [cat. «dels rahims agrests o saluatius que sapellen lambrusques $\gg$, f. $73 r]$. A esta geminación sinonímica se recurre también en el texto catalán, por lo que parece que estamos ante la referencia a la realidad, en la que anda por medio el tecnicismo llambrusca $\sim$ lambrusca, que

\footnotetext{
$8 \quad$ Este agresta es adjetivo; no ha de pensarse, por lo tanto, en su continuidad con el sust. lat. medieval agresta, ae (Du Cange: $s . v$.); se trata de adj. clásico agrestis, que encontramos todavía en Isidoro (1778: 742) aplicado a la vid: «Labrusca est vitis agrestis, quae in terrae marginibus nascitur». Se emplea aquí el latinismo agresta como adjetivo, con la terminación femenina propia del aragonés: «la vid saluage o agresta». Así lo explican, para Fr. Vicente de Burgos, Sánchez de Herrero (2008: 353; 2009b: 297, n. 53), aplicado a las peras, y Sánchez de Herrero \& Jiménez Ríos (2008: 365), aplicado a las uvas. Uva agresta está en el Glos Esc., aragonés (Castro 1936: 155, s. v. agresta, y \$1127), donde, por cierto aparece también el lat. labrusca: «La[n]brusca-e, por aceytuna, o uva mal madura».
} 
puede llegar por sí solo a utilizarse para expresar esa realidad: «Toman la flor de la vua es a saber de aquellas que fazen lambruscas» (f. 185v) [no logro dar en el manuscrito catalán con el pasaje].

El comportamiento del traductor es el mismo que el de Fr. Vicente de Burgos (1494), que para traducir del latín «Vitis agrestis est labrusca» acudió también a lambrusca: «La vid saluage o agresta es una lambrusca», f. 250r, apoyándose para su traducción en el aragonés (Sánchez González de Herrero 2008: 363 y ss.; 2009: 63; Grande López 2014: 92), aunque tampoco hubo de serle ajeno el hecho de que apareciera la voz en latín. No sería la primera vez en que un aragonesismo bajomedieval empleado en castellano estuviera aliado con el latín, que es lo que pudo ocurrir con la penetración -bastante débil- de lambrusca en castellano. Más adelante, con la traducción que hizo Laguna del Dioscórides (1555: 505 y 506), se toma labrusca directamente del texto latino que traduce, sin que haya motivos para suponer que se apoyara para ello en uno de los romances a los que vengo refiriéndome aquí ni que la palabra se hubiera institucionalizado en nuestra lengua: «De la vid salvaje [opuesta a la vid doméstica] o labrusca: Hállanse dos especies de labrusca», y continúa diciendo que esta vid salvaje se esfuerza en complacernos «principalmente quando cumple resfriar y restriñir algún miembro: las quales facultades se hallan mucho más efficazes en la labrusca [...] que en la vid cultivada. Suélense mezclar los razimos y las flores de la labrusca en el mosto». En realidad el latín propicia este término internacional, casi un tecnicismo de la viticultura, que estaba bien instalado en este pequeño Mediterráneo por el que navegaba nuestra lengua, que se extendía, aparte del catalán, por el fr. lambruche o lambrusque (TLF: s. v.; Rozier 1785: 224: «Lambruche ou lambrusque: On donne ce nom à la vigne devenue sauvage et qui croît dans les buissons»), por el occ. labrusca y lambrusca (S.-J. Honnorat 1847: $s$. $v v$.) y por el it. lambrusca (TLIO: s. v. Lambrusca, registrada desde el siglo XI).

Es natural que se terminara acudiendo a esta voz en castellano, en distintas ocasiones, aunque no terminara prendiendo en él. En el $C D H$, aunque se registra en Fr. A. de Guevara (1521) y Quevedo (b1597), aparece, sobre todo, como término propio de la viticultura. Esto explicaría que Alonso y Ruyzes de Fontecha (1602) siga empleando la voz acompañada de vid saluaje o de vba agria, o que Juan Álvarez Guerra traduzca del diccionario de Rozier (1797: 290): «Por este medio se prepara un vinagre de un sabor muy agradable con las flores de lambrusca o vid silvestre», y que, entre los pocos datos que el $C D H$ proporciona de esta palabra, los botánicos C. de Azcárate y Fernández (1893) y B. Lázaro Ibiza (1896) se refieran a la labrusca como a una variedad silvestre de la vid. El hecho es que ya J. Quer explicaba que la labrusca (que encontraba en Laguna) era la vid o parra silvestre o salvaje (1784: 487), y luego al tratar en su texto de ella recurría a vid salvaje. Este ha sido un caso 
en que el cruce entre la designación común de una palabra en determinadas lenguas y el recurso a un término especializado con aspiración de ser general en todas ellas ha dado lugar a problemas, como el que señalan Esteban Collantes \& Alfaro (1855: 271), por cierto, no demasiado conscientes de los fueros de la lengua común:

Algunos han creído que la especie de vid cultivada, vitis vinifera, era una mejora, obtenida por el cultivo de la especie borrosa a que dan los botánicos modernos el nombre de vitis labrusca [...]. La palabra labrusca, que emplea Linneo como adjetivo para designar una clase de vid indígena de la América septentrional, les ha inducido a creer que con esta especie [...] tiene mucha analogía el labrusca de los antiguos, que es la planta espontánea de la vid cultivada, causando con esto gran confusión en la nomenclatura. Y hasta en los departamentos meridionales de Francia se conoce todavía la vid silvestre con el nombre de labrusca o lambrusca.

Da la impresión de que lambrusca es un aragonesismo que procede del catalán, que, apoyado en el latín, logra introducirse como tecnicismo en español, en la forma labrusca.

\section{BROCADA'TROZO DE SARMIENTO QUE SE DEJA EN UNA CEPA PARA QUE DÉ FRUTO'}

De brocada' 'trozo de sarmiento o esqueje que con dos o tres yemas se deja en una cepa para que crezca y dé fruto al año siguiente', solo he dado con dos ejemplos, ambos en plural, en el primero de los cuales se mantiene la terminación catalana en -es:

Las brocades que hombre dexa en las çepas que son criadas en manera de parras non las deue hombre fazer mucho çerca del sarmjento viejo o duro [...] las brocadas que faras en medio del sarmjento ..., f. 69v [cat. brocades, f. 36r, en ambos casos].

El cat. brocada, derivado de broc, se extiende por una gran parte del dominio catalán, incluida Valencia (DAlcMoll: s. vv. broc y brocada; y DECAT: s. v. broc); Cavanilles (1797: 250) lo da como propio de la huerta de Alicante: «Al podar las cepas dexan en cada una tres ramos, que allí llaman brocades y cada brocada dos yemas».

Brocada se documenta también en el ámbito aragonés. El $C D H$ solo registra este ejemplo de la traducción de Palladio. El fichero general de la Academia extien- 
de la voz, a través del $A L E A N R$, por distintos puntos de las provincias de Huesca y Teruel, así como se registra en Murcia, a través de los diccionarios de A. Sevilla (1919: s. v. brocada) y de García Soriano (1932), y en el extremo oriental de Granada, recurriendo a la información de Alcalá Venceslada (1951 [no en la 1. a edición de 1934]) y del ALEA (broká), 1961, lám. 193, mapa 190.

Datos a los que añadiría los de Gargallo (1987: 557), aparte de los que encontramos en un par de tratados de silvicultura, de Elgueta Vigil (1761) y de García Sanz (1861 y 1863). Aquel (al que A. Sevilla, a quien he citado antes, utilizó como fuente), no siendo murciano de nación, sino atencino, escribe en sus «Motivos para escribir esta obra», que lo hacía «animado de la experiencia de quarenta años de práctica en esta ciudad de Murcia»; y este confesaba (1863, en su prólogo, p. V [en realidad estaba ya en la primera edición, de 1844]) que había «sido también de grande provecho el trato con los labradores y la observación continua de sus labores en los reinos de Murcia y Valencia, puntos en que la agricultura se halla tal cual adelantada». Ahí están los datos que leemos en este último:

Algunos cultivadores y plantadores de viñas, parras y parrizales creen que no sirve para planta el sarmiento que no lleve a su extremidad inferior una cachompa más o menos grande, es decir un pedazo de cepa o brocada (1861: 105).

El ingerto de escudete es para árboles hechos, en lo alto de sus brocadas o del tronco mocho, de especies de corteza fina (1863: 151).

Algún uso hubo de tener, sin embargo, en la lengua técnica castellana de la agricultura, pues lo encontramos en la traducción que hizo Álvarez Guerra de F. Rozier $(1797,1803)$ :

Brocada (pulgar, punta): Con este nombre se designa aquel sarmiento de la vid, al cual al podarle, se dejan pocas yemas. Si se quiere tener un brazo o sarmiento fuerte, se poda un grueso todo lo más bajo que sea posible, y de ese modo se llenarán los espacios vacíos con la madera que produzca.

Sin embargo, no justifica este dato suficientemente la aparición de esta palabra en los diccionarios. En este caso no se ha incluido en el de la Academia, pero sí en el de Terreros (1786) y en algunos más. Este tipo de obras, cuya fuerza prescriptiva es grande, produce el espejismo de que existe una cierta autarquía en las lenguas, capaces de bastare a sí mismas, sin contar con las demás, de forma que quien encuentre el sust. brocada en un diccionario difícilmente sospechará que está prendido con alfi- 
leres en el universo lexicográfico, sin sospechar que pudiera haber entrado allí como un aragonesismo, sino que disimula esa condición convirtiéndose en una especie de cripto-aragonesismo, que solo perciben los iniciados (los filólogos). Y esto lo hace posible su apariencia morfológica, que es la de una voz tradicional castellana, a pesar de que, si la analizamos, no encontramos en nuestra lengua una forma paralela al cat. broc que fuera su base de derivación.

Brocada parece, en fin, un préstamo del aragonés, donde a su vez había entrado procedente del catalán.

\section{ENTRECAVAR 'REMOVER LA TIERRA'}

El cat. entrecavar 'remover la tierra' (DECAT: s. v. cavar, que se registra, siguiendo al DCVB: s. v. entrecavar, en 1565, es decir con posterioridad a las dos versiones de la Agricultura de que me estoy sirviendo aquí ${ }^{9}$ ), sinónimo de palafangar, aparece indistintamente en el texto catalán y en el castellano. En catalán se trata de la voz usual para este significado de 'remover la tierra'; a ella recurre el traductor, espoleado normalmente por el cat. entrecauar, pero también sin que haya mediado esa presión: «las vjñyas que son estadas cauadas o descubiertas deuran ser entrecauadas e cobrir las rrayzes $»$, f. $138 \mathrm{v}$ [cat. «les vinyes que son stats cauats deuras magencar e cobrir els raels», f. 67r] , si suponemos, lo que me parece probable, que el manuscrito catalán que utilizaba el traductor tuviera realmente magencar.

Permitía esta palabra hacer algunas precisiones de interés para el cultivo de la tierra, tal y como vemos a continuación a través de la traducción:

En ella se matiza que hay ocasiones en que se ha de entrecavar muy por encima: «ser entrecauadas fuert ligerament es a saber que non fazen a cauar muy fondo», f. 76r [cat. «deuen esser entrecauades molt llaugerament, ço es que no fan a cauar fondo», f. 39r], lo cual se facilita utilizando los instrumentos adecuados, como vemos en «Los rrosales antigos se deuen en aqueste tiempo entrecauar con açadas chicas $\mathrm{E}$ con semblantes açadas destrales que son agudos a dos cabos y deuelos hombre podar...», f. 79r [en este caso no sigue al pie de la letra a nuestro manuscrito catalán, que presenta aquí «Los rosers antichs se deuen en aquest temps entrecauar y deulos hom podar...», f. 40v]. Hay distintas formas de entrecavar, como la que se percibe en este ejemplo: «tu los entre-

9 Lo cual acontece varias veces más, como mujada, que se documenta por primera vez en el DECAT (s. v. mode), en 1459. En otros casos puede no registrarse en ese diccionario, como ocurre con el cultismo culturar 'cultivar', que no se recoge, s. $v$. coldre. 
cauaras por tal manera que el açadon entre mas fondo que non son sus rrayzes E leuantaras asuso la tierra $\gg$, f. $83 \mathrm{v}$ [cat. «tuls entrecauaras per tal manera quel cauech entre mes fondo que no son les raels y sospendras en amunt la terra $\gg, f$. $43 r]$, frente a la acción más ligera que muestra este otro: «De entrecauar ligeramente las vjñyas nueuas», f. 155v [cat. «De magencar o cauar llaugerament les vinyes novelles», f. 73v]. Incluso la acción puede consistir simplemente en limpiar un espacio de yerbas: «E faras lo entrecauar o ljmpiar de otras yeruas», f. 130v [cat. «faraslo entrecauar daltres erbes», f. 63v], de forma que en vez de «entrecauar»se recomienda que «non se quiere entrecauar mas que las yeruas que se fazen en torno dellas que sean arrancadas con las manos», f. $107 \mathrm{r}$ [cat. «nos vol entrecauar sino que les erbes que son entorn sien arrancades», f. $53 \mathrm{v}$; si bien puede ocurrir «que las yeruas que seran nasçidas çerca dellos sean arrancadas e entrecauadas despues», f. 112r [cat. «que les erbes que naxeran prop de ells sien arrancades despres», f. 56r].

La mayor parte de los 37 ejemplos que el $C D H$ proporciona de entrecavar procede de la traducción de la Agricultura; a ellos se han de añadir dos más que están en el mismo manuscrito 10211 de la BNE: el Tratado de plantar... y la Memoria de las labranzas, que acoge también el $D I C C A X V$. Junto con estos textos técnicos de la agricultura se ha de contar con uno más, que en este pasaje tiene esa misma condición, por paradójico que parezca, La donzella Theodor (en una versión en que, por lo que se verá, hemos de sustituir la fecha de 1250 del $C D H$ por la de 1500): «En el mes de enero siendo vieja la luna deues alimpiar las arboles que pierden la foja e es tiempo despuesto para trasplantar, enxerir, cauar las viñas [e] los rosales e raer e entrecauar la alfalfa e boluer los baruechos e plantar qualquier generacio de ligumes», f. 6r, en un pasaje que está tomado de una traducción aragonesa de Andrés de Li (1492), que trabajaba para los Hurus (Rivera y Rogers 2000: p. 42, n. 32; cfr. Martos 2014: 156, nn. 7 y 8, y 181).

Los restantes ejemplos modernos del $C D H$ están escorados hacia el oriente: uno de 1962, Índice del catálogo de la XXII feria oficial y nacional de muestras de Zaragoza: «Maquinas de entrecavar remolacha»; trece de Magda Ródenas (1964), en un libro publicado en Barcelona. Del sur de esta área oriental proporciona ese corpus dos referencias al madrileño de nación, pero murciano de adopción, Javier Fuentes y Ponte (1872), quien no considera la palabra un barbarismo, y la define: «Limpiar de yerbas los bancales de hortalizas», y otro de Azorín (1903 [1913]: 386): «Un labriego va entrecavando la tierra dura».

También los datos de Google nos sitúan casi exclusivamente en esta área oriental: así tenemos en un documento de 1405 de Valvanera (La Rioja): «Dos açadones e dos açadiellas de entrecavar» (González Bachiller 2004: s. v.) y de su permanencia 
en la Rioja es prueba su uso en Matute (García Turza 1975: 49): «Entrepicar, entrecavar, hacer la entrepica o hacer la entrecava 'escardar cuando el cereal está poco crecido'» (definidos entrecava y entrecavar en pág. y 121). S. de Rojas Clemente (1818: 433), que era de Titaguas, estudió en el seminario de Segorbe y luego en Valencia, escribe: «La rebina común ceñida a solo entrecavar o recortar y ahuecar la tierra »; G. Anechina (1901: 12), que era aragonés y trata de asuntos de Aragón, en una adición a la Agricultura de Alonso de Herrera se refiere a: «Entrecavar la tierra después de la recolección, sin tocar con la azadilla los tallos». Modernamente aparece entrecavar en Segorbe (Torres Fornés 1903: 261; en realidad procede de Borao, vid. prólogo de S. Giner a Llatas 1959: 19): «Limpiar de tierra la hortaliza»; en Puebla de Hijar (Jordana y Monpeón 1916, apud Aliaga Jiménez 1999-2000: 367): «Labor ligera hecha generalmente con azada para realzar las plantas y quitar las malas hierbas que hay entre aquellas» (ahí mismo el sust. entrecava: «Labor de cavar hecha entre plantas»); y en Caspe (del caspolino Rais 1917, apud Aliaga Jiménez 1999-2000: 422): «Limpiar de hierbas la hortaliza. || 2. recargarlas con la azada poniendo alrededor del tallo tierra desmenuzada y abundante», de ahí procede la definición de Barceló Caballud, caspolino también (2011: s. v.): «Limpiar de hierbas, con la azada, los surcos y caballones de un campo. Recargar con tierra los tallos de las hortalizas plantadas». En el artículo de una revista publicada en Estella cuyos autores trabajan en Zaragoza (Cirujeda Ranzenberger \& Carlos Zaragoza 2005: 49) leemos: «Se ofrecen diferentes tipos de aperos para remover el suelo o incluso entrecavar los árboles si se requiere».

Otro grupo de ejemplos de entrecavar procede de textos en español escritos en territorios de lengua catalana, como un informe de T. Domínguez \& J. de la Croix (1808: 273) de Valencia: «De esta suerte se puede entrecavar con más facilidad», en Benacira (Valencia): «Esta operación [escardar] la llamábamos allá abajo entrecavar», (J. R. y S. 1863: 63) y el profesor y arqueólogo barcelonés J. Maluquer de Motes (1984: 165), escribe: «El hallazgo de esta tumba se realizó con motivo de entrecavar un olivo».

Dispongo de muy pocos datos explícitamente castellanos de entrecavar: está en el Diccionario de Autoridades (1732), que lo define: «No cavar mui hondo sino a medio cavar», que no toma de Nebrija, sino - lo dice textualmente- del Vocabulario del P. Alcalá; lo cual no ha de sorprendernos, pues no aparece en la primera edición del Diccionario de Nebrija, sino en una posterior (de 1513 o de 1514), quien pudo haberlo introducido ahora de P. de Alcalá. Este, puestos a hacer conjeturas, podría haber conocido la voz en Granada, hasta donde pudo llegar siguiendo el mismo camino de otros aragonesismos a los que me he referido antes. Los contados ejemplos que puedo añadir tampoco hacen que me desprenda de la inseguridad en que me 
muevo: el más antiguo con el que he logrado dar, a través de Google, aparece en un documento de 1548, del occidente de la provincia de Guadalajara (Camarero 2005: 146): «Los olivos [...] que se an de entrecavar »; luego, el Fichero General de la Academia, junto a bastantes casos aragoneses que no voy a citar, pero que refuerzan considerablemente los que he allegado antes, proporciona (aparte de la referencia al diccionario de Nebrija, de 1514) un par de ellos netamente castellanos, pero procedentes de obras técnicas, como es el caso de Villarreal Berriz (1736: 140): «Quando naciere se tendrá cuidado de quitar las yervas y entrecabarlo curiosamente quando crezca un poco» o de Colmeiro (1859: en el apartado «Términos de botánica y jardinería más usuales», 441): «Entrecavar, dar una labor ligera con el azadón». Junto a ellos he dado en Google con el siguiente de México: «El 21 de junio se inició la floración, y el 23 se entrecavaron y recalzaron, excepción de la parcela $\mathrm{V}$, que no se hizo más que entrecavar» (Boletin de Agricultura, Ingeniería e Industrias, México 1898, 24).

La introducción de este cripto-aragonesismo, que no se reputa como tal en el diccionario, lo facilitaba su apariencia morfológica - más aún que en brocada -: era predecible en castellano, pues partía ahí de la misma base del catalán y aragonés ( $c a-$ var) y de unas posibilidades formativas idénticas también (entre- + verbo); aparte de que desde el punto de vista semántico permitía establecer una gradación con respecto al verbo cavar, con el que se podía relacionar genéticamente (condición de la que carecen escardar y layar, a las que se suele acudir para explicar esta acción en español).

\section{PALAFANGAR 'REMOVER LA TIERRA'}

De palafangar 'remover la tierra', sinónimo de entrecavar, solo aparece un ejemplo en la traducción de Palladio: «Las vjñyas e los parrales deue hombre cauar e palafangar en aqueste mes de febrero en los lugares empero que son çerca de mar», f. $78 \mathrm{v}$ [cat. «les vinyes y les parrals deu hom cauar y palafangar», f. 40v]. Se trata en catalán de un derivado de palafanga 'laya' (DCVB: s. v. palafanga; DECAT: s. v. pala). Igual que su sinónimo entrecavar, permite esta palabra, tanto en el texto catalán como en el castellano, precisar el hecho de que no se extrae la tierra de un lugar, sino solo que se la remueve.

El término aragonés (a su vez préstamo del catalán) no se ha incorporado al castellano (en este ni en otros sentidos con que se emplea en catalán); ni siquiera aparece en el diccionario académico. Se encuentra en el CORDE y en el DICCA XV, pero avalado solo por este ejemplo que acabo de citar. $Y$ no abre la puerta a que pensáramos en un uso normal castellano encontrárnoslo en C. Montiu (1820: 254), pues lo da como catalán: «Aquella especie de labor que llamamos layar, y en catalán palafangar». 


\section{PÁmpano 'HoJa de la VID'}

Hay casos de palabras que, semejantes al castellano por su significante en aragonés y catalán, se diferencian por su significado, como ocurre con el cast.pámpano y el cat. o arag. pampol. El primero se refiere al 'sarmiento tierno', mientras que el segundo (junto con el occ. pampol, el fr.pampre, el it.pámpano) designa la 'hoja de la vid' (DECH: s. v. pámpano; DECAT: s. v. pampol); de ese modo el castellano se separa de este amplio espacio lingüístico situado al Oriente, relegando ese significado de 'hoja de la vid' al femenino pámpana.

En nuestra traducción se acude al cast.pámpano, pero con el significado del cat. pampol 'hoja de la vid', como vemos en los siguientes ejemplos, que pasaron al $C D H$, una parte de los cuales aparece también en el DICCA XV:

«Non deue hombre tirar los panpanos de las vjñas en los lugares calientes e secos e avn temprados», f. 10v [cat. «no deu hom lleuar los pampols de les vinyes en los llochs calents ne sechs nec encara temprats», f. 5v].

«la çepa se esfuerça de lançar pampanos o tallos de la verga vieja », f. 71v [cat. «lo çep sesforça de llançar brot de la verga vella», f. 37r].

«aquel brot o pampano todos tiempos fara su poder en fazer pampanos o ramas e non fructo», f. $71 \mathrm{v}$ [cat. «aquell brot tostemps mostra son poder en fer pampols y rames y non fruyt $\gg$, f. $37 \mathrm{r}]$.

«Las çepas o sarmjentos que podresçen las vuas tu les deues tirar los pampanos [...] E solamente dexaras los polzims susanos que defienden las vuas del sol e de la pluuja e de la frior», f. 175r [cat. «Als çeps o sarments que podreixen els rahims tuls deus lleuar los pampols a cascun costat [...] e solament leixaras los pampols sobirans que defensen los rahims de la fredor», f. 81v].

«habundan mucho en panpano los quales nasçen e se fazen en lugar fuerte duro de la parra o de la çepa », f. $64 \mathrm{r}$ [cat. «ço es que abunden molt en pampols los quals naixen his fan en lloch dur de la parra del çep», f. 33v].

«poco fructo faran Ca Antes lo conuertiran todo en rramas e en pampanos», $\mathrm{f}$. $69 \mathrm{v}$ [cat. «poco fruyt farien ans ho convertirien tot en rams y pampols», f. 36r] «deue ser despampanada es a saber que le sean tirados vna partida de los pampanos [...] Empero si el lugar do sera la vjñya es lugar caljente e seco non deues tirar los pampanos», f. 162r [cat. «deu esser espampanada ço es que sia lleuada una partida de la multitud dels pampols empero si lo lloch on sera la vinya [...] es lloch calt y sech souint deus llevar los pampols», f. 76r y v]. Un poco más adelante «despampanar las vinyas», 162r [cat. «lo espampanar de les vinyes», f. 76v]. 
$\ll$ E deyuso del vaso meteras de los pampanos en que non aya agua njn rroçio [...] E en cada vn vaso lleno tu meteras los pampanos de suso que sean frios», 190v [cat. «dauall en lo vexell posaras dels pampols en que no aia aygua ni ros [...] tu posaras aquells vexells en lloch fret y sech», f. 86r].

Da la impresión, pues, de que se ha incorporado un aragonesismo semántico al texto, en cuanto que pámpano se emplea con el significado que pampol tenía en catalán y aragonés, 'hoja de parra'.

Despampanar sigue el mismo camino que pámpano: de los varios ejemplos que encuentro, el siguiente, que acabo de citar, muestra claramente su significado de 'quitar hojas de las parras': «Deue ser despampanada es a saber que le sean tirados vna partida de los pampanos por tal que la calor del sol pueda mas ligeramente madurar la vendjmja Empero si el lugar do sera la vjñya es lugar caljente e seco non deues tirar los pampanos», f. 162 r.

De los aproximadamente 350 casos de pámpano del $C D H$ (que son una parte de los que están lematizados bajo esa forma), varios tienen en la Edad Media el significado de 'sarmiento tierno', pero también unos cuantos se emplean con el de 'hoja de parra'; entre ellos, al menos la mitad, pertenecen a textos en los cuales aparecen catalanismos y aragonesismos, como los del marqués de Villena (1427), Guido de Cauliaco (1493), Fr. Vicente de Burgos (1494) o la traducción de Tirante el Blanco (1511). Paralelamente despampanar 'quitar las hojas a las vides' vuelve a aparecer en Cauliaco (1493) y en Fr. Vicente de Burgos (1494).

Con estos datos podríamos suponer que pámpano tuvo en castellano, al principio, el significado de 'sarmiento tierno', al que ya en la Edad Media se le añadió el propio del catalán y aragonés, que terminó prendiendo en nuestra lengua. A partir del Siglo de Oro esta nueva acepción se impuso, de tal forma que a lo largo de los siglos XVII al XX se fue abandonando la tradicional, al menos en el ámbito de la literatura, ya que resistió más en los textos técnicos de agricultura, como es el caso de Gabriel Alonso de Herrera (1513) o de Gregorio de los Ríos (1592). El DECH (s. v. pámpano) daba cuenta del significado moderno de 'hoja de parra' en español por medio de un ejemplo de J. Valera (tomado del diccionario de Pagés) y de su introducción en la edición del DRAE de 1884.

Sin embargo la contaminación de sentido no afectó a la familia completa, pues despampanar (y despampanante) solo aparecen en sentido figurado, salvo en dos obras. La primera, el Diccionario de Núñez de Taboada (1825), mantiene el significado relacionado con el originario de pámpano en castellano, ya que de deslechigar remite a despampanar (que define como 'quitar las yemas o renuevos de los árboles') y a deslechugar (que define, en cambio, como 'quitar los pámpanos y ramas que na- 
cen de nuevo en la vid'). La segunda, un texto técnico de Casildo de Azcárate sobre los insectos (1894), donde señala solo el significado moderno de despampanar 'quitar las hojas a la vid': «Despampanar las vides demasiado frondosas».

\section{ESPLEYTO Y ESPLEYTAR}

He dejado para el final — pronto se verá por qué- las voces espleito y espleitar, que no han entrado en castellano. Su significado se expresa en nuestra lengua por medio de cosech a y cosechar o, dándole un sentido más general, a través de explotación o aprovechamiento y explotar o aprovechar. Estos son los ejemplos de nuestro texto:

«Los espleytos de la tierra », ff. 1r, 3v [en el primer caso, no encuentro una voz catalana a la que traduzca; en el segundo «esplets de la terra $»$, f. 2 r] .

«el campo que avra pasado por vn añyo que non y avra avido espleyto», 191bis-r [cat. esplet, f. 87r].

«plantando e expleytando aquella », f. 2 r [cat. espletant, f. 2 r]

«vn jornal de bueyes de tierra grasa espleytan .vj. mujgs de fauas», f.191 bis-r [cat. «un jornal de bous de terra grassa complexen .vj. mujgs de faues», f. 87r]

En el mismo MS 10211 de la BNE en que está esta traducción tenemos los ejemplos siguientes, que tomamos del DICCA XV:

«Suyo tendran todo en bienes sedientes segunt la suma de sus bienes rendas espleytes e emolumentes de aquellos lo metan en aquella mano», Ordinaciones (1418), f. $11 \mathrm{r}$.

$\ll$ E el senyor rey ni sus predecessores no han hauido njngun vtil spleyt nj proueyto de la dita casa e la propriedat de aquella no vale», Fondo Sástago (1453), f. 1.

«aquel judicio que por la dita causa scomençareys de los quales fruytos et spleytes que recebido e cogido haureys de los dichos lugares suso specialados et conffrontados», Fondo Sástago (1488), f. 80.

A ellos podemos añadir bastantes más de espleit y espleitar en textos aragoneses: aparte de los casos de esple(i)tar que proporciona el $D E C A T$ (s. v. esplet), se registra ya, con varios sentidos, en un amplio número de documentos, a partir de los del Alto Aragón editados por T. Navarro (Lagüéns 1992-1993: 49; vid. particularmente la n. 10). El CDH da unas cuantas referencias más de espleitar: Fueros de Aragón 
(1247-1305 [cód. finales del siglo XIII-principios del siglo XIV, Rodríguez Molina \& Octavio de Toledo 2017: 15]); documento de Irache (1385); Modificaciones de las Ordenanzas de Zaragoza (1429-c1458); así como espleitar en el escritor aragonés Jaime Huete, Comedia Viridiana (1535); y espleytar y espleyte en la traducción del Corán aljamiado (1606), de base aragonesa (López-Morillas 1982: 44-45); para esplet el DECH (s. v. esconce) nos lleva, de una manera indirecta, al siguiente ejemplo del Racontamiento d'Alixandre, texto aljamiado aragonés de mediados del siglo XVI (Vespertino Rodríguez 1987-1989: 1422 y 1432, n. 21): «Ya bos a llegado lo k’an esconçado de mi los rreyes de toda la tierra, lo que m’an dado de los presentes i de pagar los espletes».

Espleitar y espleit abundan en la documentación navarra, empezando por los Fueros de Navarra (que cito por R. Jimeno Aranguren 2016). Contamos así en el Fuero General de Navarra (1250-1279 [manuscrito de principios del siglo XIV) con 13 ejemplos de espleytar $(35,42,91,92)$; espleytar está también en el Fuero extenso de Tudela, (1247 y 1271 [copias de los siglos XIV y XVI; R. Jimeno Aranguren 2016: 434]); Fuero de Viguera y Val de Funes (siglo XIII [copia de la segunda mitad del siglo XV, según Ramos Loscertales, o del 1..$^{\text {er }}$ cuarto del siglo XVI, según Faulhaber]), espleytar y espleytes, pp. 516 y 522. Y siguiendo por los documentos (Ruiz San Pedro 1998 y 1999), en códices, la mayor parte de ellos originales y unos pocos copias de fecha muy cercanas a las de los originales, más de una docena de ejemplos que van de 1362 a 1365, en combinaciones como espletar los molinos o los bienes o tener e espleytar; está también en un documento (1423) de Carlos III, rey de Navarra, que entrega a su nieto don Carlos sus posesiones y los vasallos que están en ellas «para que los tenga, possida y espleite y defienda, como cosas suyas propias» (Monreal Zia y Jimeno Aranguren 2008: 172). Espleitamiento (tomado del $C D H$ ) aparece en el Vidal Mayor (c1250) [según Tilander, su editor; 1260-1280, en PhiloBiblon]: «En aqueill aynno dé muito frujto et se siegue daynno por tal grant espleitamiento».

Esta voz tan común en occitano abunda en el romance provenzal cispirenaico: de varios casos me conformaré con referirme a «les espleyz» en el MS $S$ del Fuero extenso de Pamplona ( $p 1343$ ) (Jimeno Aranguren 2016: 325; Monreal Zia \& Jimeno Aranguren 2008: 986).

Si destaco esta voz de las anteriores es porque su cronología en los textos que he conseguido allegar es más antigua y abundante que las palabras a que me he referido antes (de $\$ 1$ a $\$ 9$ ). Es lo que me lleva a considerar que este aragonesismo incrustado en la traducción no ha entrado en navarro-aragonés desde el catalán, sino que se trata de una palabra patrimonial en ambos espacios lingüísticos. El DECAT (s. v. esplet), por la peculiaridad cronológica y la situación corográfica de la voz, le da un trato particular: «Ha entrat molt mes endins de l'Alt Aragò i des de molt antic», 
que me he atrevido a matizar en el sentido de que ciertamente EXPLICITARE no dejó descendencia popular en castellano y sí en occitano y catalán, pero en navarro-aragonés también (lo cual no impidió que en ocasiones se tomara la forma esplet del catalán).

\section{Conclusiones}

Me he atrevido a triar unos pocos aragonesismos de este texto de finales de la Edad Media, traducido del catalán, que es, de cuantos conozco, uno de los que más catalanismos contiene. Unos aragonesismos ( $\$ 1$ conrear, $\$ 2$ empeltar, $\$ 3$ borró), catalanismos a su vez, que no se lograron introducir en español ${ }^{10}$. Otros sí lo lograron, pero introducidos como tecnicismos más que como voces propias de la lengua común; en un caso ( $\$ 5$ lambrusca) apoyándose en el latinismo; son varios aquellos en que el aragonesismo ha tenido un uso esporádico como término especializado en la jerga agrícola ( $\$ 6$ brocada, $\$ 7$ entrecavar) o no han logrado saltar a ella ( $\$ 8$ palafangar). Un tipo particular de aragonesismo es aquel en que el significante de la voz castellana incorpora a esta el significado de la voz aragonesa, lo que se conoce como un falso amigo: sería este un caso en que - a diferencia de muchos de los anteriores- el falso amigo habría terminado por imponerse en español, convertido en un amigo de verdad ( $\$ 9$ pámpano). Se termina esta relación con un aragonesismo $(\$ 10$ espleyto $\sim$ espleytar), que no llegó a penetrar en castellano, pero que tiene la particularidad de poderse explicar como voz surgida en aragonés paralelamente al catalán, como palabra patrimonial, no como préstamo tomado de esta última lengua.

De esta selección aleatoria de voces aragonesas que aparecen en esta traducción castellana de los primeros años del siglo XV o de los últimos del XIV, ciertamente la mayor parte de ellas no prendió en español. Pero la importancia del aragonés no hemos de medirla solo por los aragonesismos que se introdujeron en castellano, sino también por el hecho previo de que no dudaran los traductores en servirse de ellos, a sabiendas, incluso, de que muchos no iban a pervivir. Es la prueba de una actitud abierta hacia las lenguas de la Corona de Aragón, a las que dirigían su mirada los escritores castellanos: no solo por la importancia política y militar de la Corona, sino también porque se desarrollaba allí una cultura que para Castilla tenía una impor-

10 A ellos se pueden añadir bastantes más que he tenido que dejar de lado, como es el caso de exorc (exorch) -ca, (-qua) exorchs -es 'estéril, exorqueza 'esterilidad', fueron a su vez en aragonés préstamos del catalán. Es lo que ocurre también con ese triar que acabo de utilizar, a sabiendas de que no debió emplearse en español general, por más que, aparte de su aparición en esta traducción lo encontremos también en algún diccionario y tenga acomodo en un área cercana al valenciano, en la forma trigar: «Escoger, entresacar. En val. triar» (Llatas 1959: 205). 
tancia semejante a la que después iba a tener la italiana. De esa cultura formaba parte un humanista como Margrit y Pau, y en ella surgieron empresas editoriales como la de los Hurus, que acogieron una serie de traducciones de obras que podemos considerar que por entonces estaban en la vanguardia, mientras circulaba una cantidad de manuscritos italianos, de Dante o Boccaccio, por ejemplo, que era impensable en la Castilla del S. XV. Aunque hubiera bastado con referirme a la relevante literatura catalana del Cuatrocientos.

No es sorprendente la abundancia de traducciones de distintas lenguas al castellano que se hicieron entonces, para las que el catalán sirvió de intermediario. Quienes se dedicaban a estas tareas, lo normal es que procedieran de estos territorios del oriente peninsular, que contaran, por tanto, con un buen conocimiento del catalán y del aragonés y hasta que actuaran en su trabajo con un sentido de afirmación hacia sus lenguas. Aparte de que esas traducciones, como he sugerido al principio, no tenían por qué ser definitivas.

Otra cosa es que esto ocurra a la vez que un grupo importante de escritores aragoneses ponía su empeño en acercar todo lo posible sus usos a los propios que, por simplificar, llamaré del castellano oficial. Pero esta es otra cuestión.

\section{Bibliografía}

Agud, Manuel y Antonio Tovar (1994): «Materiales para un diccionario etimológico de la lengua vasca XXI», Anuario del Seminario de Filología Vasca "Julio de Urquijo", 28, pp. 915-992.

Alcalá Venceslada, Antonio (1951): Vocabulario andaluz, Madrid, Real Academia Española.

ALEANR = Alvar, Manuel (dir.); Llorente, Antonio y Tomás BuesA (colabrs.) (1979-1983): Atlas Lingüistico y Etnográfico de Aragón, Navarra y la Rioja, 12 vols., Zaragoza, Diputación Provincial de Zaragoza.

Aliaga JiméneZ, José Luis (1999-2000): «Documentos lexicográficos del Estudio de Filología de Aragón (II)», Archivo de Filología Aragonesa, 16, pp. 337-442.

Anechina, Gregorio (1901): El azafrán. Guia práctica para el cultivador y el negociante, s. 1. [Madrid], s. n. [Imprenta Libertad].

Azorín [José Martínez Ruiz] (1913 [1903]): Antonio Azorín. Pequeño Libro en que habla de la vida de este peregrino señor, Madrid, Renacimiento.

Barceló Caballud, Rafael (2011): Vocabulario caspolino, Zaragoza, Institución Fernando el Católico. 
BuesA, Tomás (1955): «Terminología del olivo en el alto aragonés de Ayerbe», Miscelánea filológica dedicada a monseñor Griera, Barcelona, CSIC, vol. I, pp. 57-109.

Butiñá, Julia (1996): «Sobre el prólogo de Ferrer Sayol al De re rustica de Palladio», Epos, 12, pp. 207-228.

Cabrera, Ramón (1837): Diccionario de etimologías de la legua castellana, obra póstuma publicada por Juan Pedro Ayegui, 2 vols., Madrid, Imprenta de don Marcelino Calero.

Camarero, José M. ${ }^{a}$ (2005): «La agricultura en Albalate de Zorita , Cuadernos de Etnología de Guadalajara, 37, pp. 119-172.

Cancionero de Coimbra: Cancionero de Coimbra de la Biblioteca Geral da Universidade de Coimbra, MS 1011 [citado a través del DICCAXV].

Cavanilles, Antonio José (1797): Observaciones sobre la historia natural, geografía, agricultura, población y frutos del reyno de Valencia, Madrid, Imprenta Real.

Cirujeda Ranzenberger, Alicia y Carlos Zaragoza Larios (2005): «Maquinaria para controlar la flora arvense sin químicos (y II)», La fertilidad de la tierra. Revista de agricultura ecológica, 22, pp. 48-51.

Colmeiro, Miguel (1859): Manual completo de jardineria, 3 vols., Madrid, Librerías de don Ángel Calleja.

Corán aljamiado: Traducción castellana de un morisco anónimo del año 1606, introd. de Juan Vernet, transcripción y nota preliminar de Lluis Roqué Figuls, Barcelona, RABL, 2001.

DECAT = Coromines, Joan amb la collabració de Joseph Gulsoy y Max CAHNER (1980-2001): Diccionari etimológic i complementari de la llengua catalana, 10 vols., Barcelona, Curial Edicions Catalanes.

$D E C H=$ Corominas, Joan con la colaboración de José Antonio PAscual (19801991): Diccionario critico etimológico castellano e hispánico, 6 vols., Madrid, Gredos.

Diccionario de Autoridades = Real Academia Española (1726-1739): Diccionario de la lengua castellana... En línea: <http://web.frl.es/DA.html> [29/07/2020].

$D L E=$ ReAl ACADEMIA Española (29/07/2020): Diccionario de la lengua española. En línea: <https://dle.rae.es/> [29/07/2020].

Domene Verdú, José Fernando (2010): «Caracterización lingüística del habla de Villena (Alicante)», Archivo de Filología Aragonesa, 66, pp. 217-243.

Domínguez, T. y Joaquín de la Croix (1802): «Extracto de un informe presentado a la Sociedad Económica de Valencia », Seminario de agricultura y artes dirigido a los párrocos, Madrid, Real Jardín Botánico, vol. 12. 
La donzella Theodor = Historia de la donzella Theodor, Toledo, Pedro Hagembach, 1500.

Du Cange = Du CANGE, Charles et al. (1883-1887): Glossarium mediae et infimae latinitatis. En línea: <http://ducange.enc.sorbonne.fr/> [29/07/2020].

Elgueta Vigil, Antonio de (1761): Cartilla para la agricultura de moreras y Arte para la cría de la seda, Madrid, Imprenta de don Gabriel Ramírez.

Esteban Collantes, Agustín y Agustín Alfaro (1855): Diccionario de agricultura práctica y economía rural, 7 vols., Madrid, Imprenta de Constantino Armesto.

Fondo Sástago = Pergaminos aragoneses del conde de Sástago, Arxiu de la Corona d'Aragó [citado a través del DICCA XV].

Fuentes y Ponte, Javier (1872): Murcia que se fue, Madrid, s. n. [Imprenta de la Biblioteca de Instrucción y Recreo].

García de Santa María, Gonzalo, Vida de santos = Gonzalo García de Santa María (trad.) (c1488): Vida de los sanctos religiosos de Egipto, Zaragoza, Juan Hurus [citado a través del DICCA XV].

García SANZ, José (1861): Manual de agricultura dedicado al hijo del cultivador, Madrid, Librería de don José Cuesta.

García SANZ, José (1863): Manual de selvicultura práctica, Madrid, Viuda e hijos de don José Cuesta.

García Soriano, Justo (1932): Vocabulario del dialecto murciano, Murcia, Editora Regional de Murcia.

García Turza, Claudio (1975): Matute y su léxico (Logroño), Logroño, Diputación Provincial.

Gargallo, José Enrique (1987): Una encrucijada lingüistica entre Aragón, Valencia y Castilla: El Rincón de Ademuz, Tesis doctoral inédita, Universitat de Barcelona.

Gómez Ortín, Francisco Javier (2019): Lorquinencias, Murcia, Instituto Teológico de Murcia.

GoNZÁLEZ BACHILleR, Fabián (2004): «Voces relativas a la flora y la agricultura en documentos riojanos medievales $S . X V »$, Berceo, 246, pp. 67-87.

Grande López, Clara (2014): «Cuestiones de traducción medieval: De las propiedades de malenconía de la enciclopedia De proprietatibus rerum de Bartolomé Ánglico», en Clara Grande López, Leyre Martín Aizpuru y Soraya Salicio Bravo (coords.): Con una letra joven. Avances en el estudio de la historiografia e historia de la lengua española, Salamanca, Universidad de Salamanca, pp. 91-98.

Hauf I Valls, Albert G. (1989): «Dues versions iberoromàniques de l'Opus agriculturae de Pal.ladi. Petita mostra lexicogràfica», en Günter Holtus, Georges 
Lüdi y Michael Metzeltin (eds.): La Corona de Aragón y las lenguas románicas. Miscelánea de homenaje para Germán Colón, Tübingen, Narr, pp. 383-393.

Honnorat, Simon-Jude (1847): Dictionnaire provençal-français ou dictionnaire de la langue d'oc ancienne ou moderne, 2 vols., Digne, Repos.

IDÁÑEz, Faustino (2015): Léxico de la región prebética: Limites del lenguaje andaluz $y$ del murciano, Murcia, Universidad de Murcia.

IsIDoro (1778): Operum divi Isidori. Etymologiarum, liber Primus, Matriti, Bartholomaei Ulloa.

Jimeno Aranguren, Roldán (2016): Los fueros de Navarra, Madrid, Boletín Oficial del Estado.

Labores $=$ Memoria de las labranças, MS 10211 , BNE, ff. 210r-218r, en que se copia también la traducción de Palladio [citado a través del DICCA XV].

LAGÜÉNS, Vicente (1992-1993): «Precisiones sobre el significado de algunas voces registradas en documentos altoaragoneses medievales», Archivo de Filología Aragonesa, 48-49, pp. 47-98.

Laguna, Andrés (1555): Pedazio Dioscorides anazarbeo. Acerca de la materia medicinaly de los venenos mortiferos, Anvers, Juan Latio.

LEVy, Emil (1920): Petit dictionnaire ancien occitan (provençal)-français, Heidlberg, Carl Winter.

LI, Andrés de (1492), Repertorio de los tiempos, Burgos, Pablo Hurus.

Llatas, Vicente (1959): El habla de Villar del Arzobispo y su comarca, 2 vols., Valencia, Institución Alfonso el Magnánimo.

Llorente Maldonado de Guevara, Antonio (1991): «Fronteras lingüísticas internas en territorio aragonés», en Tomás Buesa y José María Enguita (eds.): I curso de geografía lingüistica de Aragón, Zaragoza, Institución Fernando el Católico.

López Morillas, Consuelo (1982): The Qur'an in Sixteenth-Century Spain; Six Morisco versions of Sura 79, London, Tamesis Books.

Maluguer de Motes, Joan (1984): La necrópolis de la loma de Peinado, Casilla de Martos (Jaén), Barcelona, Universitat de Barcelona.

Martí Escayol, Maria Antònia (2009): «"Com vol empaltar, dues coses deu hom guordar”. La traducció en català del tractat d'agricultura de Gottfried de Francònia », Estudis d'história agrària, 22, pp. 131-164.

Martínez Romero, Tomàs (2008): «Del Llibre de Pal.ladi al Libro de Paladio: A propósito de las traducciones iberorromances medievales del Opus Agriculturae y de su autoría », Romance Philology, 62, pp. 111-136.

Martos, Josep Lluís (2014): «La editio princeps del Repertorio de los tiempos de Andrés de Li: el proyecto editorial y la recuperación del incunable», en Marta 
Haro y José Luis Canet (eds.): Texto, edición y público lector en la Universidad de Valencia, Valencia, Universitat de València, pp. 155-186.

Monreal Zia, Gregorio y Roldán Jimeno Aranguren (2008): Textos histórico-jurídicos navarros, vol. I, Historia antigua y medieval, Pamplona, Fondo de Publicaciones del Gobierno de Navarra.

Montiu, Cristóbal (1820): «Memoria en que se describe un nuevo arado y reja...», en Memorias de agricultura y artes, que se publican de orden de la Junta nacional de gobierno del comercio de Cataluña, junio de 1820, pp. 245-256.

Morrás, María (2002): «El debate entre Leonardo Bruni y Alonso de Cartagena: las razones de una polémica», Quaderns. Revista de Traducció, 7, pp. 33-57.

NúÑEZ DE TABOADA, Melchor (1825): Diccionario de la lengua castellana, 2 vols., Paris, Seguin.

Ordinaciones = Ordinaciones de Zaragoza, MS 037 de la BNE, con registros de la Cancillería sobre el gobierno de la ciudad de Zaragoza [citado a través del DICCAXV].

PASCUAL, José Antonio (2018): «La relación entre los manuscritos 10202 y 10203 de la BNE, del Árbol de las batallas», Anuario del Seminario de Filología Vasca "Julio de Urquijo", 52, pp. 647-656.

QUER, Joseph (1784): Continuación de la flora española, Madrid, Joaquín de Ibarra. R., J. y S. (1863): Las veladas de Benacacira o conversaciones sobre la agricultura y sus productos, Valencia, Imprenta de la Opinión.

Recetario de Gilberto = MS II 3063 de la Biblioteca de Palacio de Madrid [citado a través del DICCAXV].

Rivera, Isidro J. y Donna M. Rogers (2000): Historia de la donzella Teodor, Binghamton, Binghamton University.

Ródenas, Magda (1964): ABC de las plantas de jardinería y terraza, Barcelona, Gassó.

Rodríguez Molina, Javier y Álvaro Octavio de Toledo y Huerta (2017): «La imprescindible distinción entre texto y testimonio: El CORDE y los criterios de fiabilidad lingüística», Scriptum Digital, 6, pp. 5-68.

Rojas Clemente, Simón de (1818): [adición a] Gabriel Alonso de Herrera, Agricultura general, 2 vols., Madrid, Imprenta Real.

RozIER, François (1785): Cours complet d'agriculture théorique pratique, économique et de médecine rurale et vétériaire ou dictionnaire raisonné et universel d'agriculture, 6 vols., Paris, Rue et Hôtel Serpente.

RozIER, François (1797-1803): Curso completo o diccionario universal de agricultura teórica, práctica y económica, de medicina rural y veterinaria, traducido por Juan Álvarez Guerra, 16 vols., Madrid, Imprenta Real. 
SánChez de Arévalo, Rodrigo (1941): Espejo de la vida humana, Zaragoza, Hurus.

SÁnChez GonzÁlez de Herrero, M. Nieves (2008): «Deproprietaibus rerum. Versiones castellanas», Cahiers de recherches médiévales et humanistes, 19, pp. 349-366.

SÁnchez González de Herrero, M. ${ }^{a}$ Nieves y Enrique Jiménez Ríos (2008): «El proceso de la traducción castellana de fray Vicente de Burgos del De proprietatibus rerum a partir del análisis léxico», en Javier San José Lera (dir.); Francisco Javier Burguillo López y Laura Mier Pérez (eds.): La fractura historiográfica: las investigaciones de Edad Media y Renacimiento desde el Tercer Milenio, Salamanca, SEMYR, pp. 409-425.

SÁnchez GonzÁlez de Herrero, M. ${ }^{a}$ Nieves (2009a): «Traducciones castellanas de enciclopedias medievales», en Eva Martha Eckkrammer (ed.): La comparación en los lenguajes de especialidad, Berlin, Frank \& Timme GmbH, pp. 59-67.

Sánchez González de Herrero, M. ${ }^{a}$ Nieves (2009b): «La adaptación del léxico botánico en las versiones castellanas de De proprietatibus rerum », Revista de Investigación Lingüistica, 11, pp. 287-305.

Sebastián Torres, Raimon (2014): Ferrer Sayol, traductor de Pal.ladi, Tesis doctoral inédita, Universitat de Barcelona.

Sevilla, Alberto (1919): Vocabulario murciano, Murcia, Sucesores de Nogués.

TLF = ImBs, Paul (dir.) (1971-1994): Trésor de la langue française, Paris, Gallimard, 16 vols. En línea: <http://atilf.atilf.fr> [29/07/2020].

TLIO: Tesoro della lingua italiana delle origine. En línea: <http://tlio.ovi.cnr.it/ TLIO/> [29/07/2020].

Torreblanca, Máximo (1976): Estudio del habla de Villena y su comarca, Alicante, Instituto de Estudios Alicantinos.

Torres Fornés, Cayetano (1903): Sobre voces aragonesas usadas en Segorbe, Valencia, Tipografía Moderna.

Vespertino Rodríguez, Antonio (1987-1989): «Una aproximación a la datación de los manuscritos aljamiados-moriscos», Estudios Románicos, 5, Homenaje al profesor Luis Rubio García, Murcia, vol. II, pp. 1419-1439.

Villarreal Berriz, Pedro Bernardo (1736): Máquinas hidráulicas de molinos y herrerias de los árboles y montes de Vizcaya, Madrid, Antonio Marín.

Fecha de recepción: 10 de agosto de 2020 Fecha de aceptación: 17 de septiembre de 2020 